\title{
Circulating microRNA's as a diagnostic tool for hepatocellular carcinoma in a hyper endemic HIV setting, KwaZulu-Natal, South Africa: a case control study protocol focusing on viral etiology
}

K. Sartorius ${ }^{1,2,3}$, B. Sartorius ${ }^{1,3^{*}}$, A. Kramvis ${ }^{4}$, E. Singh ${ }^{5}$, A. Turchinovich ${ }^{6,7}$, B. Burwinkel ${ }^{6}$, T. Madiba ${ }^{3}$ and C. A. Winkler ${ }^{8}$

\begin{abstract}
Background: A wide range of studies has investigated the diagnostic proficiency of extracellular microRNAs (miRNAs) in hepatocellular cancer (HCC). HCC is expected to increase in Sub-Saharan Africa (SSA), due to endemic levels of viral infection (HBV/HIV), ageing and changing lifestyles. This unique aetiological background provides an opportunity for investigating potentially novel circulating miRNAs as biomarkers for HCC in a prospective study in South Africa.

Methods: This study will recruit HCC patients from two South African cancer hospitals, situated in Durban and Pietermaritzburg in the province of KwaZulu-Natal. These cases will include both HBV mono-infected and HBV/HIV co-infected HCC cases. The control group will consist of two (2) age and sex-matched healthy population controls per HCC case randomly selected from a Durban based laboratory. The controls will exclude patients if they have any evidence of chronic liver disease. A standardised reporting approach will be adopted to detect, quantify and normalize the level of circulating miRNAs in the blood sera of HCC cases and their controls. Reverse transcription quantitative polymerase chain reaction (RT-qPCR) will be employed to quantity extracellular miRNAs. Differences in concentration of relevant miRNA by case/control status will be assessed using the Wilcoxon rank-sum (Mann-Whitney $\mathrm{U})$ test. Adjustment for multiple testing (Bonferroni correction), receiver operating curves (ROC) and optimal breakpoint analyses will be employed to identify potential thresholds for the differentiation of miRNA levels of HCC cases and their controls.

Discussion: Although there is a growing base of literature regarding the role of circulating miRNAs as biomarkers, this promising field remains a 'work in progress'. The aetiology of HBV infection in HCC is well understood, as well as it's role in miRNA deregulation, however, the mediating role of HIV infection is unknown. HCC incidence in SSA, including South Africa, is expected to increase significantly in the next decade. A combination of factors, therefore, offers a unique opportunity to identify candidate circulating miRNAs as potential biomarkers for HBV/HIV infected HCC.
\end{abstract}

Keywords: Hepatocellular carcinoma, miRNA, Biomarker, Diagnosis, Staging, HBV, HIV

\footnotetext{
* Correspondence: sartorius@ukzn.ac.za

K. Sartorius and B. Sartorius are joint first authors.

'Department of Public Health Medicine, School of Nursing and Public Health, University of KwaZulu-Natal, Durban 4041, South Africa

${ }^{3}$ UKZN Gastrointestinal Cancer Research Centre (GICRC), Durban, South Africa

Full list of author information is available at the end of the article
} 


\section{Background}

MicroRNAs (miRNAs) are (mostly) endogenously developed fragments of single stranded non-coding RNA (19-25 nucleotides) that regulate more than $50 \%$ of all cell specific protein translation. The deregulation of miRNAs is linked to cancer because they play a role in modulating target genes responsible for cell proliferation, apoptosis, DNA repair, invasion and metastasis [1]. The sensitivity of miRNA expression (transcription) alteration in cancer incidence is underlined by the location of their parent genes, often found in fragile chromosomal regions that exhibit DNA amplification, deletions and translocations which deregulate miRNA expression [2]. Circulating miRNAs have been proposed as promising biomarkers for cancer pathologies because of their abundance in sera, as well as their stability under extreme conditions $[3,4]$. Serum miRNAs are resistant to ribonuclease digestion because they are protected in protein complexes or in membranous micro-vesicles that transport them in the circulatory system $[1,5]$. The stability of cell free, circulating miRNAs is underlined by the fact that successful quantification has been observed in samples stored up to 10 years at -80 degrees $C[1,6]$. Despite considerable investigation of extracellular miRNAs, the use of miRNAs as biomarkers of cancer is still regarded as a 'work in progress' and mostly restricted to research programs $[5,7]$. Continuing technological developments, however, like 2nd generation sequencing, as well as a better understanding of the pathobiological role of miRNAs, underline their future promise as clinical biomarkers [7, 8].

A wide range of studies has investigated the diagnostic proficiency of circulating miRNAs in liver diseases, including hepatocellular carcinoma (HCC), chronic hepatitis $(\mathrm{CH})$, non-alcoholic fatty liver disease (NAFLD), liver toxicity, cirrhosis and non-alcoholic steatohepatitis [9-12]. The strong causal association between HCC and $\mathrm{CH}$ continues to influence $\mathrm{HCC}$ incidence $[3,13]$ while emerging studies explain the biological role of viral miRNAs [14]. Sub-Saharan Africa, and South Africa in particular, is an endemic region for both HBV and HIV infection, as well as rapid urbanization and lifestyle changes [15]. The aim of this study is to investigate circulating miRNAs as biomarkers for HCC in South Africa against a background of both HIV/HBV mono and co-infection.

\section{Empirical evidence of circulating miRNAs in HCC}

In hepatocellular carcinoma (HCC), a range of miRNAs is deregulated in response to cancer cells that promote aberrant expressions in their target genes [16]. HCC deregulates the expression of circulating miRNAs (upwards or downwards) to inversely influence the expression of target mRNAs/ specific genes involved in cell cycle regulation, apoptosis, DNA repair, invasion and metastasis [2]. In HCC development, the miRNA mediated expression of mRNA can have either oncogenic effects or promote a loss of tumor suppressor function $[2,14,16]$.

Emerging evidence indicates multiple miRNAs are deregulated in HCC. A recent reviewed collated a wide range of studies to collectively indicate 55 miRNAs that are down-regulated and 41 miRNAs that are upregulated in HCC [16]. The presence and proficiency of circulating miRNA as biomarkers for HCC, have been tested both individually, as well as in selected groups. Examples of deregulated circulating miRNAs, identified in numerous studies, include miR-10a, miR-21, miR-23a/b, miR-25, miR-26a/b, miR-122, miR-125b, miR-192, miR-222, miR-223, miR-342-3p, miR-375, miR-423, miR-801, miR-885-5p, and miR-Let-7f [3]. It has been suggested that miR-122a is the most abundant miRNA in hepatocytes [9], that it is a reliable marker of viral infection [17] and it is down-regulated in $\sim 70 \%$ of HCC [18]. MiR-500 is also abundantly expressed in liver cancer cell lines and deregulation of miRNA occurs in $\sim 45 \%$ of HCC cases [19].

\section{HCC, viral infection and circulating miRNAs}

Viruses encode their own sets of miRNA which are used to control the expression of their host's genes [20]. The ability of a virus to package its own miRNAs into exosomes and transport them to non-infected cells was first demonstrated by the EBV virus [21]. Both viral transcripts and proteins can affect host miRNA expression, which can modulate viral and/or host protein expression [22]. MiRNAs can bind to viral genomes or transcripts and regulate viral infection and, conversely, viral infection (e.g. HIV/HCV) can modulate host-cell microRNA machinery [23]. The role of miRNAs in viral infection is being demonstrated in an increasing number of studies. MiR-122, for instance, down-regulates HBV replication by binding to the viral target sequence [24] and, conversely, binds to the HCV genome to increase viral translation and replication [25-27]. MiR-199a and miR210 bind to different sites on mRNA coding of HBsAg, reducing HBsAg expression in HepG2 2.2.15 cells [28]. MiR-15b has also been shown to modulate HBV replication by targeting the hepatocyte nuclear factor $1 \alpha$ (HNF1 $\alpha$ ) [29], while miR-130a expression is increased in $\mathrm{HCV}$ infection.

Two review papers, summarizing a wide range of studies [2, 3], identified a marker group of seven circulating miRNAs, including miR-122, miR-192, miR-223, miR-21, miR-26a, miR-27a, miR-801 that were able to distinguish between HCC, HBV, cirrhosis and healthy controls, as well as identify HCC tumor stages. Others have shown that serum levels of miR-10a and miR-125b were lower in $\mathrm{HBV}$ infected HCC patients than in chronic hepatitis 
B (CHB) patients and that a triplet of circulating miRNAs [namely miR-375, miR-25, miR-Let-7f] were able to diagnose HCC with $~ 98 \%$ accuracy [3]. Circulating miR-21 was also higher in HCC than chronic hepatitis patients and healthy controls; furthermore, its levels correlated with miR-21 expressed in HCC tumor tissue and it had better diagnostic sensitivity than alpha fetoprotein (AFP) [2, 3]. In another study, it was found that serum miR-21, -122 , and -223 were higher in $\mathrm{HCC}$ and $\mathrm{CH}$ versus controls, whereas miR-122 and miR-21 were higher for $\mathrm{CH}$ than HCC but not miR-223 [30].

\section{Biological relevance of deregulated miRNAs in HCC}

Various studies are increasingly beginning to explain the biology of specific circulating miRNAs and their potential role in HCC, with respect to cell proliferation, angiogenesis and metastasis (see Additional file 1: Table S1). Cell proliferation in HCC is promoted by the downregulation of miR-26a, which acts as a partner with miR-195 to overcome the G1/S cell cycle blockade through the repression of E2F expression. Cell proliferation in HCC is also influenced by upregulated miRNAs (e.g. miR-21, miR-216a) that promote tyrosine kinase by downregulating the PTEN tumour suppressor protein [14]. MiR-122, for example, can inhibit angiogenesis and intrahepatic metastasis by suppressing the expression of the tumour necrosis factor- $\alpha$ - converting enzyme (TACE) [14]. The metastasis of HCC is also influenced by miR-10a which regulates ephrin-type-A-receptor-4 mediated mesenchymal transition $[14,16]$.

\section{Controversial issues in circulating miRNA research}

Recent research indicates that miRNAs are found in all cellular components, where they regulate transcription, translation, alternative splicing and DNA repair [31]. A number of unsolved issues continues to delay the use of circulating miRNAs as viable cancer/ disease biomarkers. The mechanism of their generation and possible pathways is still being investigated [31] and their biological role as messenger miRNAs in signaling, remains unclear [32, 33]. A question also remains as to whether only certain types of extracellular vesicles (e.g. exosomes) transport messenger MiRNAs and others merely transport small RNA as debris [7, 34]. In addition, the elevation of extracellular miRNAs in the blood sera of cancer patients has been attributed to general conditions like inflammation, rather than as a result of an early stage tumor [7] Another issue is that recent reviews suggest that methodological problems in many earlier studies re non standardization of sample collection, sample quality control, RNA isolation, RT-qPCR and data normalization, have rendered their findings questionable $[7,17,35]$. Finally, the ability of miRNAs to silence their target mRNAs, is also influenced by polymorphisms in their parent genes that cause small changes in the miRNA nucleotides, thus inducing a change in their ability to bind to mRNA targets [16].

\section{Methods}

Non-standard data collection and analysis have precluded the publication of many miRNA studies in high impact journals. This study will adopt the suggested checklist proposed by Kirschner et al., that covers sample collection, sample quality control, RNA isolation, RT-qPCR and data normalization [35].

\section{Study population, data/sera and sample size}

Data for the study are prospective and will be recruited from all HCC patients reporting to two South African hospitals, namely, Inkosi Albert Luthuli Central Hospital (IALCH) in Durban, KwaZulu-Natal and Greys hospital in Pietermaritzburg, KwaZulu-Natal. Prospective HCC cases will be collected from January 2017 until 2020. Each patient will complete a questionnaire that provides both demographic and lifestyle data. Each patient will be tested for HIV/HBV infection, as well as routine markers assessed for each HCC patient, including AFP and ALT/ AST. A total of 200 HCC cases is estimated in the collection period and 400 healthy controls which are age and sex matched.

\section{Blood collection for miRNA assessment}

Blood will be collected from consenting patients that are diagnosed with $\mathrm{HCC}$ in the oncology departments of the IALCH and Greys hospital. An 18-20 gauge syringe needle will be used to obtain the blood sample, which will be deposited in miRNeasy collection tubes $(1.5 \mathrm{ml}$ to $2 \mathrm{ml}$ ). Within $60 \mathrm{~min}$ of blood collection samples being taken, they will be centrifuged at $2500 \mathrm{~g}$ for $20 \mathrm{~min}$ at room temperature. Plasma supernatant will be removed and samples frozen as $500 \mathrm{ul}$ aliquots and stored at -80 deg. $\mathrm{C}$ in pre-determined pools that relate to $\mathrm{HCC}$ stage 1-1 V.

\section{Assessment of haemolysis}

The level of haemolysis will be assessed by using a spectrophotometer and samples will be classified as being haemolysed if the level of free haemoglobin $\left(\mathrm{OD}_{414}\right)$ exceeds a cut off $(0.2)$. This is important in HCC with respect to miRs $15 \mathrm{a} /-16 /-210$ [16], which are altered by the haemolysis of red blood cells.

\section{RNA isolation}

The study will use a Trizol + miRNeasy modified method to isolate miRNA from serum. Critique of different approaches indicates that non-column based purification using reagents like TRIzol or QIAzol, might be more efficient with respect to samples with a low RNA 
concentration [35]. The miRNeasy kit, however, is superior in isolating small endogenous RNA that is moderately to abundantly expressed, as well as superior with respect to isolating exogenous spike-ins (C. elegans) [35]. The isolation of RNA will commence by defrosting the serum sample on ice (only invert to mix and no vortex) and transferring 400ul of serum into a fresh tube (2 ml). 1200ul of Tri-Reagent LS (Invitrogen or Sigma) will be added and the sample vortexed, and then incubated for $5 \mathrm{~min}$ at room temperature (RT), before adding $2 \mathrm{ul} \mathrm{cel-mir-39}(5 \mathrm{fmol} / \mathrm{ul})$ and $10 \mu \mathrm{g}$ glycogen (20 $\mathrm{mg} / \mathrm{ml}$, RNA grade). The sample will be vortexed again before adding 320ul chloroform and vortexed for 5-10 s. All samples will then be vortexed (vigorously) for $45 \mathrm{~s}$ and incubated at RT for $10 \mathrm{~min}$. Samples will then be centrifuged at $16000 \mathrm{~g}$ at room temperature before carefully transferring the supernatant $(\approx 800 \mathrm{ul})$ into a fresh tube $(2 \mathrm{ml}) .1 .5$ volumes ethanol and mix will be added by pipetting up and down several times.

Each sample in 700ul aliquots will be added to a mini spin column (Qiagen miRNeasy Kit) and centrifuged at $16000 \mathrm{~g}$ for $10 \mathrm{~s}$ and the flow through discarded. The column will then be washed once with 700ul RWT Buffer, followed by a wash with 500ul RPE Buffer, and centrifuged at $16000 \mathrm{~g}$ for $15 \mathrm{~s}$. The column will be washed again by 500ul RPE Buffer and further centrifuged at $16000 \mathrm{~g}$ for $15 \mathrm{~s}$. The column will then dry spin for $1 \mathrm{~min}$ at maximum speed and be transferred to a fresh collection tube. Finally, 50ul of RNase free water will be added to the column, incubated for $2 \mathrm{~min}$ and centrifuged at 16000 for $1 \mathrm{~min}$.

\section{RNA quantification}

It is presumed that the larger sample input of 400ul will promote the detection of RNA concentration using standard spectrophotometry [35]. The RNA concentration will be measured using a Qubit RNA HS Assay kit in preference to the NanoDrop, because it is presumed that the elutes will contain contaminating proteins and polysaccharides. The Qubit kit is also specific to ssRNA.

\section{Reverse transcription real time quantitative PCR}

This process commences with the reverse transcription (RT) of total mature miRNA (10 ng) from the pooled serum in order to synthesize cDNA using a TaqMan ${ }^{\circ}$ microRNA Reverse Transcription Kit (catalogue number 4366596; Applied Biosystems) and Megaplex RT miRNA specific primers (catalogue number 4399966 from Applied Biosystems). The manufacturer's protocol will be adhered to with respect to the reverse transcription of up to approximately 380 miRNAs thus ensuring the appropriate miRNA cDNA library is developed. RT will be performed using a thermo-cycler (Mastercycler Epgradient thermocycler; Eppendorf). The following specific cycling conditions will be used; 40 cycles of $16{ }^{\circ} \mathrm{C}$ for $2 \mathrm{~min}$, followed by $42{ }^{\circ} \mathrm{C}$ for $1 \mathrm{~min}$ and then $50{ }^{\circ} \mathrm{C}$ for $1 \mathrm{~s}$. In order to de-activate the transcriptase, a final cycle at $80^{\circ} \mathrm{C}$ for $5 \mathrm{~min}$ is completed.

In order to ensure sufficient miRNA cDNA material is available for RT- PCR, cDNA libraries generated from the previous step, will be pre-amplified under supplier directions using a primer (Megaplex PreAmp primer, catalogue number 4399233; Applied Biosystems) and a PreAmp Master Mix (catalogue number 4384266; Applied Biosystems). The PreAmp primer pool selected will be based on a library of (forward) primers that have been identified for human miRNAs that mediate hepatocellular carcinoma and related cirrhotic conditions including viral infection (HBV). A universal reverse primer will be employed. The pre-amplification cycling conditions will be run under prescribed temperature and time cycles. These include a cycle at $95{ }^{\circ} \mathrm{C}$ for $10 \mathrm{~min}$, a cycle of $55{ }^{\circ} \mathrm{C}$ for $2 \mathrm{~min}$ and a cycle of $72{ }^{\circ} \mathrm{C}$ for $2 \mathrm{~min}$. This will then be followed by 12 cycles of $95{ }^{\circ} \mathrm{C}$ for $30 \mathrm{~s}$ and $60{ }^{\circ} \mathrm{C}$ for $4 \mathrm{~min}$. Finally, the samples will then be held at $99.9^{\circ} \mathrm{C}$ for $10 \mathrm{~min}$.

The expression levels of miRNA are then determined by TaqMan Low Density Arrays (TLDA). The TLDA step follows the pre-amplification of the cDNA libraries [36]. TLDA commences with the dilution of product of the previous step in RNase-free water that is combined with a gene expression master mix (TaqMan). The diluted product is transferred onto a 384-well TaqMan Low Density (TLDA) microarray plate (TaqMan Human MicroRNA Array A, catalogue number 4398965; Applied Biosystems). The microarray plate incorporates a real-time $\operatorname{TaqMan}^{\text {TM }}$ Array Microfluidic Card that has been customized to include up to 384 microarray 'hits' with primers and probes situated in each well for up to 384 miRNAs.

The supplier instruction pack will be followed for RTqPCR using a sequence detection system (ABI PRISM 7900HT-Applied Biosystems) under a specific set of cycling conditions. These conditions in sequence are $50{ }^{\circ} \mathrm{C}$ for $2 \mathrm{~min}$ and $94.5^{\circ} \mathrm{C}$ for $10 \mathrm{~min}$. The final sequences are 40 cycles of $95{ }^{\circ} \mathrm{C}$ for $30 \mathrm{~s}$ and $59.7^{\circ} \mathrm{C}$ for $1 \mathrm{~min}$. The cycle threshold, namely, the fractional cycle number at which the fluorescence passes the fixed threshold of 0.2 , will be generated by software (SDS 2.3 -Applied Biosystems). An endogenous control, Mamm U6 is embedded in the microarray (TaqMan Human MicroRNA Arrays).

\section{Normalization of RT-qPCR data (Cq values)}

The normalization of miRNA levels will be assessed using the average recovery of the spike-ins and this will be compared to the standard deviation of ubiquitous hepatocyte miRNAs like miR-122. The relative 
expression levels of miRNAs will be calculated using the comparative $\Delta \Delta C_{\mathrm{t}}$ method [37,38]. The fold changes in miRNAs will be calculated by the eq. $2_{t}^{-\Delta \Delta C}$. Cluster 3.0 software will be used to perform unsupervised hierarchical clustering, using Pearson's correlation metrics and average linkage methods. Java Treeview 1.1 .3 will be used to visualize the clustering results.

\section{Statistical analysis}

All statistical calculations were performed using Stata 13.0 and/or R. The Wilcoxon rank-sum (Mann-Whitney $\mathrm{U})$ test or Kruskal-Wallis test will be used to assess differences in serum concentration levels of miRNA levels by group. Receiver operating characteristic (ROC) curves and will be constructed and the area under the ROC curve (AUC) calculated, along with various performance statistics (sensitivity, specificity, PPV, NPV) based on the estimated optimal breakpoint for a given miRNA that best differentiates HCC cases from controls. Parametric Linear regression analysis may be used to examine correlations between the levels of the miRNAs and a range of HCC related variables and other liver function parameters. Non-parametric equivalents will also be employed if the assumption of the parametric linear regression approach is not upheld. Interaction terms for co-infection of $\mathrm{HIV} / \mathrm{HBV}$ will be included to assess differences in miRNA profile and concentration between individuals with no infection versus discrete (singular infection) versus co-infection. A correction for multiple testing (Bonferroni correction) will be employed. An adjusted $P$ value of $<0.05$ will be considered statistically significant.

\section{Discussion}

Although there is a growing base of literature regarding circulating miRNA as potential biomarkers for cancer, this field can still be classified as 'a work in progress', largely because of the non- standardised protocols used in many earlier studies, as well as unproved hypotheses relating to the biology and role of circulating miRNAs [39, 40]. This could be especially relevant in developing settings with a high burden of oncogenic viruses (HBV/ HIV) that mediate miRNA deregulation in carcinogenesis. Given the inherent regulatory function of miRNAs, it is highly likely that many miRNAs (both host and viral), expressed in relevant tissue, influence both the biological behaviour and clinical phenotype of the tumour. The development of powerful tools for miRNA characterization and quantitation, such as qRT-PCR and Deep Sequencing, suggests that the discovery pipeline for miRNA biomarkers could potentially be more efficient than "traditional" proteomic biomarker pipelines [39]. The latter is limited by delays or bottlenecks at the antibody generation phase as well as "quantitative assay development for validation of biomarker candidates" [39].

The results from this proposed study will help identify circulating miRNAs as HCC cancer biomarkers in a setting with high $\mathrm{HIV} / \mathrm{HBV}$ co-infection. As the functional roles of miRNAs in HCC development are further elucidated and better understood, we foresee that HCC specific blood-based miRNA biomarkers will be useful in both diagnosing $\mathrm{HCC}$ at an earlier stage, as well as assisting with predicting the clinical course and/or therapeutic response to currently available therapies.

\section{Additional file}

Additional file 1: Table S1. MiRNA deregulation, targets and effect in HCC. (DOCX $12 \mathrm{~kb}$ )

\section{Abbreviations}

BREC: Biomedical Research Ethics Committee; $\mathrm{CH}$ : Chronic hepatitis; HBV: Hepatitis B virus; HCC: Hepatocellular cancer; HCV: Hepatitis C virus; HIV: Human Immunodeficiency virus; IALCH: Inkosi Albert Luthuli Central Hospital; KZN: Kwazulu-Natal; NAFLD: Non-alcoholic fatty liver disease; NASH: Non-alcoholic steatohepatitis; NPV: Negative predictive value; PLC: Primary liver cancer; PPV: Positive predictive value; ROC: Receiver operating characteristic; RT-PCR: Reverse transcription polymerase chain reaction; SSA: Sub-Saharan Africa; UTR: Untranslated region

\section{Acknowledgements}

Not applicable.

\section{Funding}

This study will be largely funded through the South African Medical Research Council [MRC] (Grant reference: MRC-RFA-CCRC-01-2014). This project will also be funded with federal funds from the National Cancer Institute, National Institute of Health, under contract HHSN26120080001E. The content of this publication does not necessarily reflect the views or policies of the Department of Health and Human Services, nor does mention of trade names, commercial products, or organizations imply endorsement by the U.S. Government. This research will be supported in part by the Intramural Research Program of the NIH, National Cancer Institute, Center for Cancer Research.

\section{Availability of data and materials}

Not applicable.

\section{Authors' contributions}

KS and BS formulated the study concept and design. AK reviewed and corrected manuscript specifically with respect to viral component. AT and BB reviewed and corrected manuscript specifically with respect to all miRNA biology and proposed method. ES, TM, CW reviewed and corrected manuscript especially with respect to HCC component of proposal. All authors contributed to the study protocol and were involved with reviewing all the iterations. All authors reviewed and approved final manuscript.

Ethics approval and consent to participate

Ethics approval for this study has been received from the Biomedical Research Ethics Committee (BREC) at University of KwaZulu-Natal (BREC Reference Number: BE059/15). All study participants will sign an informed consent form before participating in the study. Patient confidentiality will be ensured.

Consent for publication

Not applicable.

Competing interests

The authors declare that they have no competing interests. 


\section{Publisher's Note}

Springer Nature remains neutral with regard to jurisdictional claims in published maps and institutional affiliations.

\begin{abstract}
Author details
'Department of Public Health Medicine, School of Nursing and Public Health, University of KwaZulu-Natal, Durban 4041, South Africa. ${ }^{2}$ Faculty of Commerce, Law and Management, University of the Witwatersrand, Johannesburg, South Africa. ${ }^{3}$ UKZN Gastrointestinal Cancer Research Centre (GICRC), Durban, South Africa. ${ }^{4}$ Hepatitis Virus Diversity Research Unit, Department of Internal Medicine, School of Clinical Medicine, Faculty of Health Sciences, University of the Witwatersrand, Parktown, Johannesburg, South Africa. ${ }^{5}$ South African National Cancer Registry, National Health Laboratory Service, Johannesburg, South Africa. ${ }^{6}$ Molecular Epidemiology Group, German Cancer Research Centre, Heidelberg, Germany. ${ }^{7}$ SciBerg e.Kfm, Mannheim, Germany. ${ }^{8}$ Basic Research Laboratory, Centre for Cancer Research, National Cancer Institute, Leidos Biomedical Research, Inc. Frederick Nat. Lab. for Cancer Research, Frederick, MD, USA.
\end{abstract}

Received: 6 December 2016 Accepted: 14 December 2017 Published online: 28 December 2017

\section{References}

1. Kosaka N, Iguchi H, Ochiya T. Circulating microRNA in body fluid: a new potential biomarker for cancer diagnosis and prognosis. Cancer Sci. 2010; 101(10):2087-92.

2. Schwarzenbach $H$, Nishida N, Calin GA, Pantel K. Clinical relevance of circulating cell-free microRNAs in cancer. Nat Rev Clin Oncol. 2014;11(3): 145-56.

3. Enache LS, Enache EL, Ramière $C$, Diaz $O$, Bancu L, Sin A, André $P$. Circulating RNA molecules as biomarkers in liver disease. Int J Mol Sci. 2014; 15(10):17644-66

4. Gui J, Tian Y, Wen X, Zhang W, Zhang P, Gao J, Run W, Tian L, Jia X, Gao Y Serum microRNA characterization identifies miR-885-5p as a potential marker for detecting liver pathologies. Clin Sci. 2011;120(5):183-93.

5. Mo M-H, Chen L, Fu Y, Wang W, Fu SW. Cell-free circulating miRNA biomarkers in cancer. J Cancer. 2012;3:432-48.

6. Pritchard CC, Kroh E, Wood B, Arroyo JD, Dougherty KJ, Miyaji MM, Tait JF, Tewari M. Blood cell origin of circulating microRNAs: a cautionary note for cancer biomarker studies. Cancer Prev Res. 2012;5(3):492-7.

7. Witwer KW. Circulating microRNA biomarker studies: pitfalls and potential solutions. Clin Chem. 2015;61(1):56-63.

8. Etheridge A, Gomes CP, Pereira RW, Galas D, Wang K. The complexity, function, and applications of RNA in circulation. The origin, function and diagnostic potential of extracellular microRNA in human body fluids; 2014. p. 19.

9. Szabo G, Bala S. MicroRNAs in liver disease. Nat Rev Gastroenterol Hepatol. 2013;10(9):542-52.

10. Zhang Y, Jia Y, Zheng R, Guo Y, Wang Y, Guo H, Fei M, Sun S. Plasma microRNA-122 as a biomarker for viral-, alcohol-, and chemical-related hepatic diseases. Clin Chem. 2010;56(12):1830-8.

11. Bala S, Petrasek J, Mundkur S, Catalano D, Levin I, Ward J, Alao H, Kodys K, Szabo G. Circulating microRNAs in exosomes indicate hepatocyte injury and inflammation in alcoholic, drug-induced, and inflammatory liver diseases. Hepatology. 2012;56(5):1946-57.

12. Coulouarn C, Factor VM, Andersen JB, Durkin ME, Thorgeirsson SS. Loss of miR-122 expression in liver cancer correlates with suppression of the hepatic phenotype and gain of metastatic properties. Oncogene. 2009; 28(40):3526-36.

13. Zhou J, Yu L, Gao X, Hu J, Wang J, Dai Z, Wang J-F, Zhang Z, Lu S, Huang X. Plasma microRNA panel to diagnose hepatitis $B$ virus-related hepatocellular carcinoma. J Clin Oncol. 2011;29(36):4781-8.

14. Sun J, Lu H, Wang $X$, Jin H. MicroRNAs in hepatocellular carcinoma: regulation, function, and clinical implications. Sci World J. 2013;924206. http://doi.org/10.1155/2013/924206

15. Mphahlele MJ, Lukhwareni A, Burnett RJ, Moropeng LM, Ngobeni JM. High risk of occult hepatitis B virus infection in HIV-positive patients from South Africa. J Clin Virol. 2006:35:14-20.

16. Morishita A, Masaki T. miRNA in hepatocellular carcinoma. Hepatol Res. 2015:45(2):128-41.
17. Turchinovich A, Tonevitsky AG, Cho WC, Burwinkel B. Check and mate to exosomal extracellular miRNA: new lesson from a new approach. Front $\mathrm{Mol}$ Biosci. 2015;2:11

18. Gramantieri L, Ferracin M, Fornari F, Veronese A, Sabbioni S, Liu C-G, Calin GA, Giovannini C, Ferrazzi E, Grazi GL. Cyclin G1 is a target of miR-122a, a microRNA frequently down-regulated in human hepatocellular carcinoma. Cancer Res. 2007:67(13):6092-9.

19. Yamamoto Y, Kosaka N, Tanaka M, Koizumi F, Kanai Y, Mizutani T, Murakami Y, Kuroda M, Miyajima A, Kato T. MicroRNA-500 as a potential diagnostic marker for hepatocellular carcinoma. Biomarkers. 2009;14(7):529-38.

20. Laganà A, Russo F, Veneziano D, Di Bella S, Pulvirenti A, Giugno R, Croce CM, Ferro A. Extracellular circulating viral microRNAs: current knowledge and perspectives. Front Genet. 2013:4:120.

21. Laguna JC, Alegret M, Roglans N. Simple sugar intake and Hepatocellular carcinoma: epidemiological and mechanistic insight. Nutrients. 2014;6(12): 5933-54.

22. Bandiera S, Pfeffer S, Baumert TF, Zeisel MB. miR-122-a key factor and therapeutic target in liver disease. J Hepatol. 2015;62(2):448-57.

23. Piedade D, Azevedo-Pereira JM: MicroRNAs, HIV and HCV: a complex relation towards pathology. Rev Med Virol. 2016;26(3):197-215.

24. Chen Y, Shen A, Rider PJ, Yu Y, Wu K, Mu Y, Hao Q, Liu Y, Gong H, Zhu Y, et al. A liver-specific microRNA binds to a highly conserved RNA sequence of hepatitis B virus and negatively regulates viral gene expression and replication. FASEB J. 2011:25(12):4511-21.

25. Jopling CL, Yi M, Lancaster AM, Lemon SM, Sarnow P. Modulation of hepatitis C virus RNA abundance by a liver-specific MicroRNA. Science. 2005; 309(5740):1577-81.

26. Jopling CL, Schutz S, Sarnow P. Position-dependent function for a tandem microRNA miR-122-binding site located in the hepatitis C virus RNA genome. Cell Host Microbe. 2008;4(1):77-85.

27. Li Y, Masaki T, Yamane D, McGivern DR, Lemon SM. Competing and noncompeting activities of miR-122 and the $5^{\prime}$ exonuclease Xrn1 in regulation of hepatitis C virus replication. Proc Natl Acad Sci U S A. 2013; 110(5):1881-6.

28. Zhang GL, Li YX, Zheng SQ, Liu M, Li X, Tang H. Suppression of hepatitis B virus replication by microRNA-199a-3p and microRNA-210. Antivir Res. 2010; 88(2):169-75.

29. Dai X, Zhang W, Zhang H, Sun S, Yu H, Guo Y, Kou Z, Zhao G, Du L, Jiang S, et al. Modulation of HBV replication by microRNA-15b through targeting hepatocyte nuclear factor 1alpha. Nucleic Acids Res. 2014;42(10):6578-90.

30. Xu J, Wu C, Che X, Wang L, Yu D, Zhang T, Huang L, Li H, Tan W, Wang C. Circulating MicroRNAs, miR-21, miR-122, and miR-223, in patients with hepatocellular carcinoma or chronic hepatitis. Mol Carcinog. 2011;50(2):136-42.

31. Makarova JA, Shkurnikov MU, Wicklein D, Lange T, Samatov TR, Turchinovich AA, Tonevitsky AG: Intracellular and extracellular microRNA: an update on localization and biological role. Prog Histochem Cytochem. 2016;51(3-4):3349. doi:10.1016/j.proghi.2016.06.001. Epub 2016 Jun 25.

32. Turchinovich A, Weiz L, Burwinkel B. Extracellular miRNAs: the mystery of their origin and function. Trends Biochem Sci. 2012;37(11):460-5.

33. Turchinovich A, Samatov T, Tonevitsky A, Burwinkel B. Circulating miRNAs: cell-cell communication function? The origin, function and diagnostic potential of extracellular microRNA in human body fluids; 2014. p. 27.

34. Turchinovich A, Tonevitsky AG, Burwinkel B. Extracellular miRNA: a collision of two paradigms. Trends Biochem Sci. 2016;41(10):883-92.

35. Kirschner MB, van Zandwijk N, Reid G. Cell-free microRNAs: potential biomarkers in need of standardized reporting. Front Genet. 2013;4:56.

36. Turchinovich A, Burwinkel B. Distinct AGO1 and AGO2 associated miRNA profiles in human cells and blood plasma. RNA Biol. 2012;9(8):1066-75.

37. Schmittgen TD, Livak KJ. Analyzing real-time PCR data by the comparative CT method. Nat Protoc. 2008;3(6):1101-8.

38. Livak KJ, Schmittgen TD. Analysis of relative gene expression data using real-time quantitative PCR and the 2- $\Delta \Delta C T$ method. Methods. 2001;25(4): 402-8.

39. Mitchell PS, Parkin RK, Kroh EM, Fritz BR, Wyman SK, Pogosova-Agadjanyan EL, Peterson A, Noteboom J, O'Briant KC, Allen A. Circulating microRNAs as stable blood-based markers for cancer detection. Proc Natl Acad Sci. 2008; 105(30):10513-8.

40. Tsang JC, Dennis Lo Y. Circulating nucleic acids in plasma/serum. Pathology. 2007;39(2):197-207. 